\title{
A New Approach of Direct Sample-Digestion before Vaporization for Determination of a Metal in Rocks by Inductively Coupled Plasma Atomic Emission Spectrometry
}

\author{
Yasuaki OKaмoto, Reiko KIKKaWA, and Terufumi FujIWARA \\ Department of Chemistry, Graduate School of Science, Hiroshima University, \\ Higashihiroshima 739-8526, Japan
}

\begin{abstract}
A new approach to sample digestion, subsequent vaporization and introduction to an inductively coupled plasma (ICP) atomic emission spectrometer was developed for the direct determination of magnesium. To each small sample cuvette made of tungsten, a ground rock sample was precisely weighed. The cuvette was situated on a tungsten boat furnace. Ammonium fluoride solution was added to the cuvette as a chemical modifier. After the on-furnace digestion has been completed, the analyte, magnesium, in the cuvette was vaporized and introduced into the ICP atomic emission spectrometer. Since the powdered samples were wet-digested in the sample cuvettes prior to vaporization, they could be analyzed by using a calibration curve prepared from aqueous standard solutions. This method was applied to the determination of magnesium in several standard reference materials with satisfactory results.
\end{abstract}

(Received October 30, 2000; Accepted January 9, 2001)

\section{Introduction}

Inductively coupled plasma atomic emission spectrometry (ICPAES) is mainly intended for the analysis of aqueous samples. Hence, in most applications, a pneumatic nebulizer has been used to convert the aqueous sample solution into a finely dispersed aerosol. However, this introduction method allows only approximately $3-12 \%$ of the nebulized solution to actually reach the ICP. ${ }^{1}$ The value further decreases with any increase in viscosity, acid concentration or salt-content of the solution.

Sample digestion procedures are necessary to convert them into liquid form, because most of the samples analyzed are not in liquid form. Direct solid introduction schemes are very attractive from the viewpoints of increasing the transport efficiency compared with solution nebulization, analyzing small amounts of samples because of several milligram amounts requirements and not suffering from contaminations inherent in extensive sample preparation. Various approaches have been described, including the application of a graphite cup direct insertion device, ${ }^{2-6}$ a pelletized solid insertion device, ${ }^{2}$ in-torch vaporization, ${ }^{7}$ an electrically vaporized thin-film plasma technique, ${ }^{8}$ and electrothermal vaporization (ETV) techniques, ${ }^{9-12}$ as well as their modifications to a graphite furnace arc (halonarc) system, ${ }^{13}$ and to a pelletized ETV system. ${ }^{14}$

The ETV techniques combined with ICP-AES have further advantages of increased selective vaporization of the analyte from matrix compounds and of independent optimization of the ETV device and the ICP spectrometer. Several difficulties still remain, such as introducing pre-weighed small amounts of finely powdered samples into the ETV device, removing the residues from it, and finding an accurate calibration method.

In the previous work, a newly conceived ETV method, abbreviated as the furnace-fusion (FF) method, was designed, ${ }^{15,16}$ i.e., a biological solid sample was fused with diammonium hydrogenphosphate and tetramethylammonium hydroxide (TMAH) fluxes and decomposed completely on the tungsten boat furnace (TBF). Then the analyte was successively vaporized and introduced into the ICP to measure the atomic emission assigned to the analyte atom or ion. The other merit of the FF method is small sample consumption, i.e., only several milligram amounts of samples are required. This article will discuss the application of the FF method to the direct determination of magnesium, as an example, in several rock samples. The determination of magnesium in rock samples is not so difficult by any routine analytical procedure. Magnesium was nevertheless adopted as an analyte in this experiment. This is because magnesium will form a thermally stable species during the FF procedure, which will make it easier to evaluate the applicability of the proposed method. As for the fusion flux, the combined use of diammonium hydrogenphosphate and TMAH was not preferable to decompose the siliceous matrixes. As an alternative decomposition reagent, ammonium fluoride was evaluated. Kuroda et al. ${ }^{17}$ reported that the presence of ammonium fluoride served to remove severe matrix effects when cobalt and nickel in rock samples were determined by graphite furnace atomic absorption spectrometry (GF-AAS). Their method is as follows: Each aliquot of lithium carbonate and boric acid as fluxes and rock samples were placed into a platinum crucible. Then, the contents were fused to decompose and the fusion cake was dissolved in dilute hydrochloric acid. Finally, to the resulting sample solution, ammonium fluoride was added as a chemical modifier. The solution was analyzed by GF-AAS. Concerning the rapid sample preparation for the determination of silicate materials, a flow injection system based on on-line microwave-assisted digestion was demonstrated by Almeida et al. as a tool to perform silicate rock dissolution in hydrofluoric-nitric mixed acid medium. ${ }^{18}$ Compared with their method, our proposed one had excellent 
Table 1 Instrument operating conditions

\begin{tabular}{|c|c|}
\hline \multicolumn{2}{|c|}{ ICP atomic emission spectrometer (Seiko II SPS4000) } \\
\hline R. F. incident power & $1.3 \mathrm{~kW}$ \\
\hline \multicolumn{2}{|l|}{ Argon gas flow rate } \\
\hline Plasma gas & $16 \mathrm{dm}^{3} \min ^{-1}$ \\
\hline Auxiliary gas & $1.0 \mathrm{dm}^{3} \min ^{-1}$ \\
\hline Analytical line & Mg I $285.213 \mathrm{~nm}$ \\
\hline Observation height & $8.0 \mathrm{~mm}$ above load coil \\
\hline \multicolumn{2}{|c|}{ Metal furnace vaporizer (modified Seiko II SAS-705V) } \\
\hline Powdered sample & ca. $0.25 \mathrm{mg}$ \\
\hline $\mathrm{NH}_{4} \mathrm{~F}$ & $7.0 \mathrm{mg}$ \\
\hline Drying & $150^{\circ} \mathrm{C}$ for $30 \mathrm{~s}$ \\
\hline Ashing & $480^{\circ} \mathrm{C}$ for $60 \mathrm{~s}(\operatorname{ramp} 10 \mathrm{~s})$ \\
\hline Vaporization & $2400^{\circ} \mathrm{C}$ for $25 \mathrm{~s}(\operatorname{ramp} 10 \mathrm{~s})$ \\
\hline \multicolumn{2}{|l|}{ Carrier gas flow rate } \\
\hline Argon gas & $1800 \mathrm{~cm}^{3} \mathrm{~min}^{-1}$ \\
\hline Hydrogen gas & $55 \mathrm{~cm}^{3} \mathrm{~min}^{-1}$ \\
\hline
\end{tabular}

detectability of magnesium and presumably a wider range of applicability. Moreover, the use of hydrofluoric acid was avoidable, which would require the use of appropriate plastic materials instead of glassware.

\section{Experimental}

\section{Apparatus}

The experimental setup used in this experiment was described in the references. ${ }^{15,16,19}$ Briefly, a Seiko II (Chiba) Model SPS4000 ICP atomic emission spectrometer was used. The continuum background emission was automatically corrected with a wavelength modulation device. Regarding temporary signal acquisitions for the ETV technique, up to 2 elements or wavelengths were simultaneously measurable with a two channel monochromator. By means of an attached MS-DOS personal computer, integrated emission intensity (peak area) or maximum emission intensity (peak height) could be estimated for each element. A Seiko II Model SAS-705V metal furnace atomizer for AAS, equipped with a tungsten boat (U-type, $6 \mathrm{~mm}$ $\times 60 \mathrm{~mm}$ ), was used after several modifications. The sample cuvettes $(6 \mathrm{~mm} \times 15 \mathrm{~mm})$ were shaped by cutting both edges of the tungsten boats. If necessary, up to $30 \mathrm{~mm}^{3}$ of aqueous sample solution alone, or less than $20 \mathrm{~mm}^{3}$ of aqueous solution and $3 \mathrm{mg}$ of sample powder, could be placed on the cuvette. Each cuvette could be positioned on the furnace. Gilson Medical Electronics (Villiers-le-Bel, France) Model Pipetman P-10, P-20 and Microman M-10 digital pipettes were used for aqueous magnesium standard solution and reagent solution injections, respectively. A Mettler Toledo (Zürich, Switzerland) Model AT261 semi-microbalance, whose readability was 0.01 $\mathrm{mg}$, was used to weigh the sample on the sample cuvette. Solid samples were ground manually with agate mortars.

\section{Reagents}

Aqueous magnesium(II) solutions were prepared by diluting a $1000 \mathrm{mg} \mathrm{dm}^{-3}$ magnesium(II) stock solution (Atomic Absorption Standard Solution, Kanto Chemical, Tokyo) with $0.12 \mathrm{~mol} \mathrm{dm}^{-3}$ hydrochloric acid. The solid standard samples were obtained from the National Institute of Standards and Technology (NIST, Washington, D.C., USA) and the Geological Survey of Japan (GSJ, Tsukuba, Japan). A chemical modifier solution was prepared by dissolving $7.0 \mathrm{~g}$ ammonium fluoride (GR for analysis-grade, Merck, Darmstadt, Germany) with $10 \mathrm{~cm}^{3}$ of water.

\section{Recommended procedure}

An aliquot of a sample was ground to a fine powder in the agate mortar. The resulting average particle size was found to be approximately $10 \mu \mathrm{m}$ by using a particle analyzer. By means of the microbalance, each aliquot of the prepared sample ( $c a$. $0.25 \mathrm{mg}$ ) was weighed accurately into the sample cuvette, which was preconditioned by heating to expel magnesium impurities and by confirming a blank signal level. If higher detectabilities were needed, up to $3 \mathrm{mg}$ of the prepared sample powder could be weighed. The cuvette was superposed on the TBF through the sample insertion port (12 $\mathrm{mm}$ i.d.) of the glass dome with a pair of tweezers. An aliquot of the ammonium fluoride solution was added with the Microman M-10 digital pipette. For the standard batch, used for construction of a calibration curve, the cuvette was situated on the TBF. Then aliquots of $10 \mathrm{~mm}^{3}$ of chemical modifier solution and the magnesium(II) standard solution were placed into it.

The TBF was ramped through three steps for drying and ashing followed by a vaporization and introduction step. During the ashing step, the solid particles were wet-digested or dissolved with hydrofluoric acid, which was derived from ammonium fluoride by thermal decomposition. Simultaneously, a large portion of the excess ammonium fluoride was expelled together with tetrafluorosilicate through the insertion port which had been left open. After the insertion port had been closed with a silicone rubber stopper, the analyte, completely decomposed in the sample cuvette, was vaporized and introduced into the plasma emission source through the port to the torch (4 mm i.d.). The transient signals were integrated by the software attached to the spectrometer, which ran synchronously with the vaporizer. The operating conditions used in this work are summarized in Table 1.

\section{Results and Discussion}

\section{Optimization of chemical modification and/or sample decomposition reagent}

The most intense ICP excited atomic magnesium line is the Mg II $279.553 \mathrm{~nm}$ line, while the second most intense is the $\mathrm{Mg}$ II $280.270 \mathrm{~nm}$ line. These lines are commonly used for the ultra-trace and trace determination of magnesium in various samples. These two lines are too sensitive, however, to be useable for the analysis of geological samples, since magnesium is a predominant element in those samples. The Mg I 285.213 $\mathrm{nm}$ line or Mg II $279.806 \mathrm{~nm}$ line, which shows approximately one-tenth of the sensitivities of the former lines, may be more adequate for the applications in geological chemistry. Therefore, in this experiment, Mg I $285.213 \mathrm{~nm}$ line was used as the analytical line throughout.

Because of the vast difference in matrixes between solid and liquid forms, aqueous standards were not always useable for preparing calibration curves. Therefore, CRMs have most frequently been used as calibrants. In this case, the selection of the CRMs was very important, e.g., the matrixes of the CRMs should be similar to those of the samples; the analyte concentrations in the samples should be in its concentration range of the CRMs. Moreover, an important disadvantage of this method is that a blank cannot be quantified and certified. The standard addition method was effective to overcome some interferences caused by matrixes.

In our proposed method, however, each solid sample was 


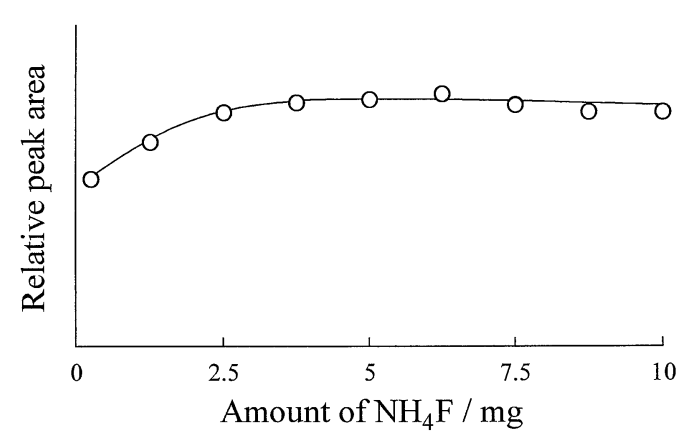

Fig. 1 Effect of ammonium fluoride on the emission intensity of magnesium. Sample, Rhyolite (GSJ JR-1). See Table 1 for other conditions.

chemically predigested during the ashing stages with hydrofluoric acid derived from the thermal decomposition of ammonium fluoride. A silicate-based matrix is corroded by fluoride-etching to be expelled as volatile tetrafluorosilicate, while the analyte reacted with fluoride ion to form thermally stable magnesium fluoride salt. Consequently, the resulting chemical species of the analyte in the ashed solid samples was the same as that in the corresponding aqueous standards.

Examinations were carried out by recording signals from each aliquot of the Rhyolite (GSJ JR-1) used as the sample. Generally, hydrofluoric acid has been successfully used for the decomposition of silicate-containing materials. However, it was not recommended since explosive reactions caused a decrease in reproducibility. In this experiment, ammonium fluoride was used. The reagent was gradually decomposed on heating and was converted to hydrofluoric acid. As Fig. 1 shows, an adequate amount of ammonium fluoride was $2.5 \mathrm{mg}$ or more for $0.25 \mathrm{mg}$ sample. In the case that no ammonium fluoride was added, the removal of the siliceous matrixes was insufficient. As a result, a large portion of matrixes was introduced into the ICP at the vaporization stage, which causes both decrease in reproducibility of measurement and instability of the plasma. Hence an amount of $7.0 \mathrm{mg}$ was selected, which corresponds to $10 \mathrm{~mm}^{3}$ of $70 \%$ ammonium fluoride solution.

\section{Effect of temperature program}

A moderate elevation of the temperature throughout the heating process was essential. The drying temperature was set to $150^{\circ} \mathrm{C}$ for $30 \mathrm{~s}$, and this was sufficient to remove the solvent of ammonium fluoride solution as a vapor.

During the ashing stage, the chemical modifier, ammonium fluoride, was subsequently degraded to produce hydrofluoric acid and ammonia. The resulting hydrofluoric acid reacted with the siliceous matrix to produce the gaseous tetrafluorosilicate, while the product as well as excess reagent was removed through the sample injection hole. The analyte also reacted with fluoride ion to form magnesium fluoride, a thermally stable compound (b.p. $2260^{\circ} \mathrm{C},{ }^{20}$ m.p. $1248^{\circ} \mathrm{C}^{20}$ ). Introduction of a large amount of fluoride should be avoided, otherwise the plasma became unstable and reproducible results were not obtainable. At $480^{\circ} \mathrm{C}$, excess modifier could be expelled as white fumes. To remove a large portion of it, $60 \mathrm{~s}$ was necessary. As Fig. 2 shows, a vaporization temperature of $2400^{\circ} \mathrm{C}$ was suitable to obtain sensitive and quantitative signals. Although further temperature elevation over $2400^{\circ} \mathrm{C}$ caused a higher signal intensity (peak height), it resulted also in the decrease in the lifetime of TBF, typically 50 firings. At the conditions adopted, the TBF was useable eternally. In order to

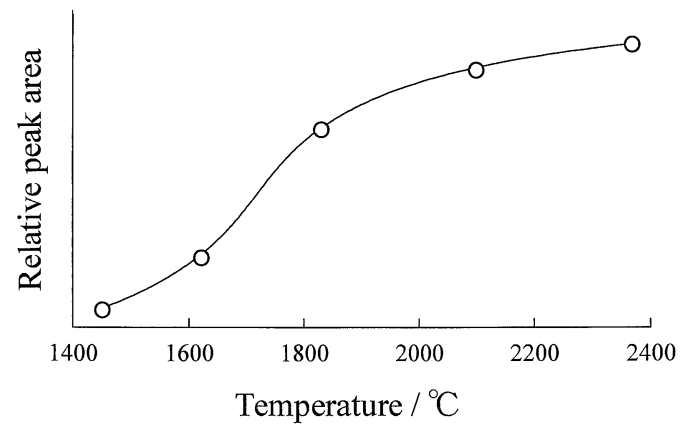

Fig. 2 Effect of vaporization temperature on the emission intensity of magnesium. Sample, Rhyolite (GSJ JR-1); $\mathrm{NH}_{4} \mathrm{~F}, 7.0 \mathrm{mg}$. See Table 1 for other conditions.

establish complete vaporization of the analyte, a hold time of 25 s was necessary.

The sensitivity for magnesium increased as the argon carrier gas flow rate increased within the tested range of 600-2000 $\mathrm{cm}^{3} \mathrm{~min}^{-1}$. Concerning the hydrogen gas, it was effective to prevent the deterioration of the TBF and the sample cuvettes. A hydrogen gas flow of $55 \mathrm{~cm}^{3} \mathrm{~min}^{-1}$ was admitted to the argon carrier gas.

\section{Basic analytical performance}

As no realistic blank samples are available, the conventional definition of the detection limit is inappropriate in the direct solid sampling. Therefore, the detection limit was estimated using an aqueous solution, which was defined as the absolute amount of magnesium required to yield a peak area that was three times the standard deviation of the blank in the presence of ammonium fluoride. The blank is a standard solution containing no magnesium(II) ion. The measurement was repeated eight times. The detection limit estimated was $1.9 \mathrm{ng}$. The detection limit could be improved drastically by measuring at $\mathrm{Mg}$ II $279.553 \mathrm{~nm}$ or at Mg II $280.270 \mathrm{~nm}$. As an alternative procedure, with a larger sample amount, a proportionally lower detection limit could be attained. Less than $3 \mathrm{mg}$ of prepared sample powder can be placed into the cuvette.

A linear calibration graph for magnesium intersecting the origin of the coordinate axes and covering absolute amounts of at least up to $500 \mathrm{ng}$ of magnesium was established. The relative standard deviation of eight replicate measurements obtained with $50 \mathrm{ng}$ of magnesium was $4.1 \%$. By using a number of exchangeable small sample cuvettes, sample throughput can be facilitated. Approximately 25 batches could be vaporized per hour.

\section{Application to practical samples}

The accuracy of the proposed method was examined. Taking into account the possibility of calibrating solids by the calibration curve method using aqueous standard solutions, magnesium was determined in several certified reference materials. The results are illustrated in Fig. 3, where the integrated signal (peak area) is plotted against the absolute amount of magnesium, calculated from both the certified value and the amount of prepared sample taken. All plots obtained with the solid samples are very close to the straight line obtained with the aqueous standard solutions. The analytical results of the Rhyolite (GSJ JR-1), Lead-Barium Glass (NIST SRM 89), and High-Alumina Sand (NIST SRM 1413) were $0.073 \pm 0.004 \%(n=4), 0.018 \pm 0.002 \%(n=4)$, and $0.036 \pm$ $0.005 \%(n=4)$, respectively, while the certified or reference 


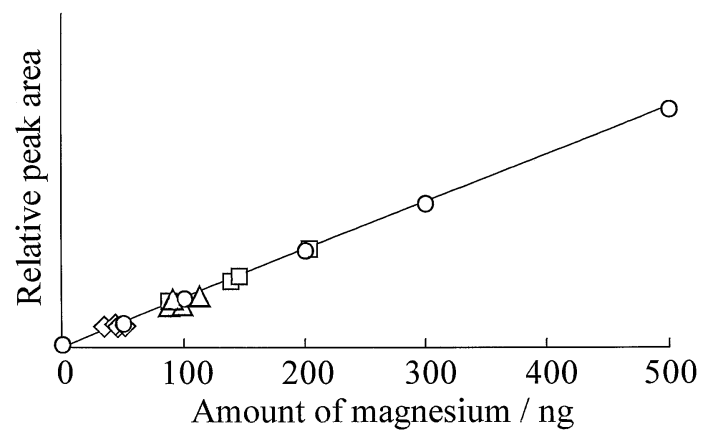

Fig. 3 Calibration curve of magnesium. O, Aqueous magnesium solution; $\square$, Rhyolite; $\diamond$, Lead-Barium Glass; $\Delta$, High-Alumina Sand.

values are $0.072,0.02$, and $0.04 \pm 0.006 \%$, respectively. Thus, it is concluded that the direct analysis of magnesium by this method is quite accurate even without matrix matching and sample pre-digestion.

In conclusion, a new FF method was designed. The rock samples were wet-digested chemically with ammonium fluoride in the small sample cuvette. During the process, the major component of siliceous matrixes was removed as tetrafluorosilicate vapor, while the analyte, magnesium, in the solid sample was selectively vaporized to introduce into the ICP apparatus at the last stage. Therefore, the tedious sample digestion procedures commonly used in the analysis of solid samples were not needed. The FF method permits standardization more easily using aqueous standard solutions. Regarding the sample throughput, this FF method makes it possible to measure a number of samples sequentially by preparing a lot of cuvettes, each containing a solid sample, prior to firing. Further efforts will be mainly focused on the other elements.
2. L. Blain, E. D. Salin, and D. W. Boomer, J. Anal. At. Spectrom., 1989, 4, 721.

3. V. Karanassios, G. Horlick, and M. Abdullah, Spectrochim. Acta, 1990, 45B, 105.

4. M. Umemoto, K. Hayashi, and H. Haraguchi, Anal. Chem., 1992, 64, 257.

5. V. Karanassios and T. J. Wood, Appl. Spectrosc., 1999, 53, 197.

6. C. D. Skimmer, M. Cazagou, J. Blaise, and E. D. Salin, Appl. Spectrosc., 1999, 53, 191.

7. H. R. Badiei and V. Karanassios, J. Anal. At. Spectrom., 1999, 14, 603.

8. K. M. Trivedi, S. W. Brewer Jr., and R. D. Sacks, Appl. Spectrosc., 1990, 44, 367.

9. I. Atsuya, T. Itoh, and T. Kurotaki, Spectrochim. Acta, 1991, 46B, 103.

10. P. Verrept, R. Dams, and U. Kurfürst, Fresenius' J. Anal. Chem., 1993, 346, 1035.

11. L. Moens, P. Verrept, S. Boonen, F. Vanhaecke, and R. Dams, Spectrochim. Acta, 1995, 50B, 463.

12. A. Golloch, M. Haversch-Kock, and F. PlantikowVoßgätter, Spectrochim. Acta, 1995, 50B, 501.

13. T. Kántor and Gy. Záray, Fresenius' J. Anal. Chem., 1992, $342,927$.

14. V. Karanassios, J. M. Ren, and E. D. Salin, J. Anal. At. Spectrom., 1991, 6, 527.

15. Y. Okamoto, J. Anal. At. Spectrom., 1999, 14, 1767.

16. Y. Okamoto, Fresenius' J. Anal. Chem., 2000, 367, 295.

17. R. Kuroda, T. Nakano, Y. Miura, and K. Oguma, J. Anal. At. Spectrom., 1986, 1, 429.

18. M. D. D. Almeida, K. C. Leandro, C. V. D. Costa, R. E. Santelli, and M. de la Guardia, J. Anal. At. Spectrom., $\mathbf{1 9 9 7 , ~ 1 2 , 1 2 3 5 . ~}$

19. Y. Okamoto, Fresenius' J. Anal. Chem., 2000, 367, 300.

20. "The Merck Index", ed. S. Budavari, M. J. O'Neil, A. Smith, and P. E. Heckelman, 11th ed., 1989, Merck, Parway.

\section{References}

1. B. L. Sharp, J. Anal. At. Spectrom., 1988, 3, 939. 